\title{
Author and Keyword Indexes for Volume 52
}

\section{AUTHOR INDEX TO VOLUME 52}

Agarwal, A., see Kumar, V.

Aguinis, H., see Steel, P.

Akella, L.Y., see Kumar, V.

Albino-Pimentel, J., Oetzel, J., Oh, C.H., Poggioli, N.A., Positive institutional changes through peace: The relative effects of peace agreements and non-market capabilities on FDI, 1256

Albino-Pimentel, J., see Georgallis, P.

Amore, M.D., Corina, M., Political elections and corporate investment: International evidence, 1775

Anand, J., McDermott, G., Mudambi, R., Narula, R., Innovation in and from emerging economies: New insights and lessons for international business research, 545

Antolín-López, R., see Montiel, I.

Arnold, D.G., Universal research ethics and international business studies, 1229

Arregle, J.-L., Chirico, F., Kano, L., Kundu, S.K., Majocchi, A., Schulze, W.S., Family firm internationalization: Past research and an agenda for the future, 1159

Arregle, J.-L., Chirico, F., Kano, L., Kundu, S.K., Majocchi, A., Schulze, W.S., Correction to: Family firm internationalization: Past research and an agenda for the future, 1199

Ault, J.K., Newenham-Kahindi, A., Patnaik, S., Trevino and Doh's discourse-based view: Do we need a new theory of internationalization?, 1394

Bartsch, F., see Mandler, T.

Belderbos, R., Grabowska, M., Kelchtermans,S., Leten, B., Jacob, J., Riccaboni, M., Whither geographic proximity? Bypassing local $R \& D$ units in foreign university collaboration, 1302

Benito, G.R.G., see Surdu, I.

Bergh, D., Research methods in international business, 1225

Berns, J.P., Gonda, M., Sellar, C., Whole country-oforigin network development abroad, 479

Bertrand, O., Betschinger, M.-A., Moschieri, C., Are firms with foreign CEOs better citizens? A study of the impact of CEO foreignness on corporate social performance, 525

Betschinger, M.-A., see Bertrand, O.
Beugelsdijk, S., see Steel, P.

Bohas, A., Morley, M.J., Kinra, A., Perlmutter revisited: Revealing the anomic Mindset, 1695

Bohnsack, R., Ciulli, F., Kolk, A., The role of business models in firm internationalization: An exploration of European electricity firms in the context of the energy transition, 824

Borah, S.B., see Kumar, V.

Bosco, F.A., see Field, J.G.

Brakman, S., Garretsen, H., van Witteloostuijn, A., Robots do not get the coronavirus: The COVID19 pandemic and the international division of labor, 1215

Brewster, C., see Minbaeva, D.

Bucheli, M., see Minefee, I.

Budhwar, P., see e Doh, J.

Carnovale, S., see Zhao, Y.

Cavusgil, S.T., see Jean, R.-J.B.

Cavusgil, S.T., see Ozturk, A.

Cerar, J., Nell, P.C., Reiche, B.S., The declining share of primary data and the neglect of the individual level in international business research, 1365

Chan, C.M., Du, J., The dynamic process of promarket reforms and foreign affiliate performance: When to seek local, subnational, or global help?, 1854

Chen, R., El Ghoul, S., Guedhami, O., Kwok, C.C.Y., Nash, R., International evidence on state ownership and trade credit: Opportunities and motivations, 1121

Chen, S., see Wang, D.

Chirico, F., see Arregle, J.-L.

Ciravegna, L., see Kano, L.

Ciulli, F., See Bohnsack, R.

Clougherty, J.A., Zhang, N., Foreign investor reactions to risk and uncertainty in antitrust: U.S. merger policy investigations and the deterrence of foreign acquirer presence, 454

Corina, M., see Amore, M.D.

Cramton, C.D., Köhler, T., Levitt, R.E., Using scripts to address cultural and institutional challenges of global project coordination, 56

Cuervo-Cazurra, A., see Montiel, I.

Currim, I.S., see Mintz, O. 
Dau, L.A., Morck, R., Yeung, B.Y., Business groups and the study of international business: A Coasean synthesis and extension, 161

Dau, L.A., see Moore, E.M.

Davies, S., see Stoermer, S.

de Jong, M., see Mintz, O.

Delios, A., see $\mathrm{Hu}, \mathrm{T}$.

Deng, Z., Liesch, P.W., Wang, Z., Deceptive signaling on globalized digital platforms: Institutional hypnosis and firm internationalization, 1096

Deng, Z., see Sun, P.

Dheer, R.J.S., Egri, C.P., Treviño, L.J., A crosscultural exploratory analysis of pandemic growth: The case of COVID-19, 1871

Ding, H., see Li, J.

Doh, J., Budhwar, P., Wood, G., Long-term energy transitions and international business: Concepts, theory, methods, and a research agenda, 951

Doh, J.P., see Sun, P.

Doh, J.P., see Treviño, L.J.

Döring, S., Drobetz, W., El Ghoul, S., Guedhami, O., Schröder, H., Institutional investment horizons and firm valuation around the world, 212

Drobetz, W., see Döring, S.

$\mathrm{Du}$, J., see Chan, C.M.

Egri, C.P., see Dheer, R.J.S.

El Ghoul, S., Guedhami, O., Kwok, C.C.Y., Zheng, Y., The role of creditor rights on capital structure and product market interactions: International evidence, 121

El Ghoul, S., see Chen, R.

El Ghoul, S., see Döring, S.

Erin Bass, A., Grøgaard, B., The long-term energy transition: Drivers, outcomes, and the role of the multinational enterprise, 807

Fainshmidt, S., see Zhao, Y.

Falaster, C., Ferreira, M.P., Li, D., The influence of generalized and arbitrary institutional inefficiencies on the ownership decision in crossborder acquisitions, 1724

Ferreira, M.P., see Falaster, C.

Field, J.G., Bosco, F.A., Kraichy, D., Uggerslev, K.L., Geiger, M.K., More alike than different? A comparison of variance explained by cross-cultural models, 1797

Fitzsimmons, S., see Minbaeva, D.

Forsgren, M., Holm, U., Complementing the Uppsala model? A commentary on Treviño and Doh's paper "Internationalization of the firm: A discourse-based view", 1407
Fortwengel, J., The formation of an MNE identity over the course of internationalization, 1069

Froese, F.J., see Stoermer, S.

Froese, F.J., Stoermer, S., Reiche, B.S., Klar, S., Best of both worlds: How embeddedness fit in the host unit and the headquarters improve repatriate knowledge transfer, 1331

Garretsen, H., see Brakman, S.

Geiger, M.K., see Field, J.G.

Genin, A.L., Tan, J., Song, J., State governance and technological innovation in emerging economies: State-owned enterprise restructuration and institutional logic dissonance in China's highspeed train sector, 621

Georgallis, P., Albino-Pimentel, J., Kondratenko, N., Correction to: Jurisdiction shopping and foreign location choice: The role of market and nonmarket experience in the European solar energy industry, 878

Georgallis, P., Albino-Pimentel, J., Kondratenko, N., Jurisdiction shopping and foreign location choice: The role of market and nonmarket experience in the European solar energy industry, 853

Gonda, M., see Berns, J.P.

Grabowska, M., see Belderbos, R.

Granqvist, N., see Ritvala, T.

Gregorič, A., Rabbiosi, L., Santangelo, G.D., Diaspora ownership and international technology licensing by emerging market firms, 671

Greve, H.R., see Surdu, I.

Grøgaard, B., see Erin Bass, A.

Guedhami, O., see Chen, R.

Guedhami, O., see Döring, S.

Guedhami, O., see El Ghoul, S.

Hagen, B., see Hennart, J.-F.

Håkanson, L., The death of the Uppsala school: Towards a discourse-based paradigm?, 1417

Han, C.M., see Mandler, T.

Han, N., see Prud'homme, D.

Hanousek, J., Shamshur, A., Svejnar, J., Tresl, J., Corruption level and uncertainty, FDI and domestic investment, 1750

Hartmann, J., Inkpen, A.C., Ramaswamy, K., Different shades of green: Global oil and gas companies and renewable energy, 879

Hejazi, W., Tang Tang, J., Wang, W., Selection, learning, and productivity at the firm level: Evidence from Canadian outward FDI, 306 
Hennart, J.-F., Majocchi, A., Hagen, B., What's so special about born globals, their entrepreneurs or their business model?, 1665

Holm, U., see Forsgren, M.

Hope, O.-K., Jiang, S., Vyas, D., Government procurement and financial statement certification: Evidence from private firms in emerging economies, 718

$\mathrm{Hu}, \mathrm{T} .$, Natarajan, S., Delios, A., Sister cities, crossnational FDI, and the subnational FDI location decision, 1279

$\mathrm{Hu}, \mathrm{y} .$, see $\mathrm{Li}, \mathrm{J}$.

Hult, G.T.M., see Samiee, S.

Husted, B.W., see Montiel, I.

Inkpen, A.C., see Hartmann, J.

Ioulianou, S.P., Leiblein, M.J., Trigeorgis, L., Multinationality, portfolio diversification, and asymmetric MNE performance: The moderating role of real options awareness, 388

Ioulianou, S.P., see Ioulianou, S.P.

Jacob, J., see Belderbos, R.

Jandhyala, S., see Wang, D.

Jean, R.-J.B., Kim, D., Zhou, K.Z., Cavusgil, S.T., E-platform use and exporting in the context of Alibaba: A signaling theory perspective, 1501

Jiang, S., see Hope, O.-K.

Johanson, J., Johanson, M., Speed and synchronization in foreign market network entry: A note on the revisited Uppsala model, 1628

Johanson, M., see Johanson, J.

Jung, H., see Westjohn, S.A.

Juntunen, J.K., see Patala, S.

Kano, L., Ciravegna, L., Rattalino, F., The family as a platform for FSA development: Enriching new internalization theory with insights from family firm research, 148

Kano, L., see Arregle, J.-L.

Katsikeas, C.S., see Samiee, S.

Kelchtermans,S., see Belderbos, R.

Kim, D., see Jean, R.-J.B.

Kinra, A., see Bohas, A.

Klar, S., see Froese, F.J.

Köhler, T., see Cramton, C.D.

Kolk, A., see Bohnsack, R.

Kondratenko, N., see Georgallis, P.

Kozlenkova, I.V., Lee, J.-Y., Xiang, D., Palmatier, R.W., Sharing economy: International marketing strategies, 1445

Kraichy, D., see Field, J.G.
Kumar, V., Borah, S.B., Sharma, A., Akella, L.Y., Chief marketing officers' discretion and firms' internationalization: An empirical investigation, 363

Kumar, V., Nim, N., Agarwal, A., Platform-based mobile payments adoption in emerging and developed countries: Role of country-level heterogeneity and network effects, 1529

Kundu,S.K., see Arregle, J.-L.

Kwok, C.C.Y., see Chen, R.

Kwok, C.C.Y., see El Ghoul, S.

Lazzarini, S.G., Mesquita, L.F., Monteiro, F., Musacchio, A., Leviathan as an inventor: An extended agency model of state-owned versus private firm invention in emerging and developed economies, 560

Lee, J.-Y., see Kozlenkova, I.V.

Leiblein, M.J., see Ioulianou, S.P.

Leten, B., see Belderbos, R.

Levitt, R.E., see Cramton, C.D.

Li, D., see Falaster, C.

Li, J., Ding, H., Hu, Y., Wan, G., Dealing with dynamic endogeneity in international business research, 339

Li, Q., see Wang, D.

Li, Y., see Tian, L.

Liesch, P.W., see Deng, Z.

List of Guest Editors and Reviewers, 1897

Lundan, S., see Patala, S.

Luo, X.R., see Wang, D.

Luo, Y., see Nambisan, S.

Magnusson, P., see Westjohn, S.A.

Majocchi, A., see Arregle, J.-L.

Majocchi, A., see Hennart, J.-F.

Malhotra, S., see Morgan, H.M.

Mandler, T., Bartsch, F., Han, C.M., Brand credibility and marketplace globalization: The role of perceived brand globalness and localness, 1559

Maznevski, M.L., see Stahl, G.K.

McDermott, G., see Anand, J.

Minbaeva, D., Fitzsimmons, S., Brewster, C., Beyond the double-edged sword of cultural diversity in teams: Progress, critique, and next steps, 45

Minefee, I., Bucheli, M., MNC responses to international NGO activist campaigns: Evidence from Royal Dutch/Shell in apartheid South Africa, 971

Mingo, S., see Moore, E.M.

Mintz, O., Currim, I.S., Steenkamp, J.-B.E.M., de Jong, M., Managerial metric use in marketing 
decisions across 16 countries: A cultural perspective, 1474

Monaghan, S., see Reuber, A.R.

Montiel, I., Cuervo-Cazurra, A., Park, J., AntolínLópez, R., Husted, B.W., Implementing the United Nations' Sustainable Development Goals in international business, 999

Moore, E.M., Dau, L.A., Mingo, S., The effects of trade integration on formal and informal entrepreneurship: The moderating role of economic development, 746

Morck, R., see Dau, L.A.

Morgan, H.M., Sui, S., Malhotra, S., No place like home: The effect of exporting to the country of origin on the financial performance of immigrant-owned SMEs, 504

Morley, M.J., see Bohas, A.

Moschieri, C., see Bertrand, O.

Mudambi, R., see Anand, J.

Nambisan, S., Luo, Y., Toward a loose coupling view of digital globalization, 1646

Narula, R., see Anand, J.

Nash, R., see Chen, R.

Natarajan, S., see $\mathrm{Hu}, \mathrm{T}$.

Nell, P.C., see Cerar, J.

Newenham-Kahindi, A., see Ault, J.K.

Nguyen, D.D., see Qi, S.

Nim, N., see Kumar, V.

Nippa, M., Patnaik, S., Taussig, M., MNE responses to carbon pricing regulations: Theory and evidence, 904

Nummela, N., see Sasaki, I.

Obadia, C., Robson, M.J., The two sides of cooperation in export relationships: When more is not better, 1616

Oetzel, J., see Albino-Pimentel, J.

Oh, C.H., see Albino-Pimentel, J.

Ozturk, A., Cavusgil, S.T., Ozturk, O.C., Consumption convergence across countries: measurement, antecedents, and consequences, 105

Ozturk, O.C., see Ozturk, A.

Palmatier, R.W., see Kozlenkova, I.V.

Pan, Y., see Tian, L.

Parente, R., Melo, M., Andrews, D., Kumaraswamy, A., Vasconcelos, F., Public sector organizations and agricultural catch-up dilemma in emerging markets: The orchestrating role of Embrapa in Brazil, 646

Parente, R., see Zhao, Y.
Park, J., see Montiel, I.

Patala, S., Juntunen, J.K., Lundan, S., Ritvala, T., Multinational energy utilities in the energy transition: A configurational study of the drivers of FDI in renewables, 930

Patnaik, S., see Ault, J.K.

Patnaik, S., see Nippa, M.

Peltokorpi, V., Pudelko, M., When more is not better: A curvilinear relationship between foreign language proficiency and social categorization, 78

Peng, Y., see Westjohn, S.A.

Piekkari, R., see Ritvala, T.

Poggioli, N.A., see Albino-Pimentel, J.

Prud'homme, D., Tong, T.W., Han, N., A stakeholder-based view of the evolution of intellectual property institutions, 773

Pudelko, M., see Peltokorpi, V.

Qi, S., Nguyen, D.D., Government connections and credit access around the world: Evidence from discouraged borrowers, 321

Rabbiosi, L., see Gregorič, A.

Rajwani, T., see Sun, P.

Ramaswamy, K., see Hartmann, J.

Rattalino, F., see Kano, L.

Ravasi, D., see Sasaki, I.

Reiche, B.S., Research handbook of global leadership: Making a difference, 1425

Reiche, B.S., see Cerar, J.

Reiche, B.S., see Froese, F.J.

Reuber, A.R., Tippmann, E., Monaghan, S., Global scaling as a logic of multinationalization, 1031

Riccaboni, M., see Belderbos, R.

Ritvala, T., Granqvist, N., Piekkari, R., A processual view of organizational stigmatization in foreign market entry: The failure of Guggenheim Helsinki, 282

Ritvala, T., Piekkari, R., Geopolitics of the knowledge-based economy, Sami Moisio Routledge, New York, 2018, 182 pp. ISBN: 978-1138821996, 334

Ritvala, T., see Patala, S.

Robson, M.J., see Obadia, C.

Samiee, S., Katsikeas, C.S., Hult, G.T.M., The overarching role of international marketing: Relevance and centrality in research and practice, 1429

Santangelo, G.D., see Gregorič, A.

Sasaki, I., Nummela, N., Ravasi, D., Managing cultural specificity and cultural embeddedness 
when internationalizing: Cultural strategies of Japanese craft firms, 245

Schröder, H., see Döring, S.

Schulze,W.S., see Arregle, J.-L.

Sellar, C., see Berns, J.P.

Shamshur, A., see Hanousek, J.

Sharma, A., see Kumar, V.

Siegel, D., see Sun, P.

Song, J., see Genin, A.L.

Stahl, G.K., Maznevski, M.L., Unraveling the effects of cultural diversity in teams: A retrospective of research on multicultural work groups and an agenda for future research, 4

Steel, P., Beugelsdijk, S., Aguinis, H., The anatomy of an award-winning meta-analysis: Recommendations for authors, reviewers, and readers of meta-analytic reviews, 23

Steenkamp, J.-B.E.M., see Mintz, O.

Stendahl, E., Stendahl, S., Tippmann, E., Control changes in multinational corporations: Adjusting control approaches in practice, 409

Stendahl, S., see Stendahl, E.

Stoermer, S., Davies, S., Froese, F.J., The influence of expatriate cultural intelligence on organizational embeddedness and knowledge sharing: The moderating effects of host country context, 432

Stoermer, S., see Froese, F.J.

Sui, S., see Morgan, H.M.

Sun, P., Deng, Z., Wright, M., Partnering with Leviathan: The politics of innovation in foreignhost-state joint ventures, 595

Sun, P., Doh, J.P., Rajwani, T., Siegel, D., Navigating cross-border institutional complexity: A review and assessment of multinational nonmarket strategy research, 1818

Surdu, I., Greve, H.R., Benito, G.R.G., Back to basics: Behavioral theory and internationalization, 1047

Svejnar, J., see Hanousek, J.

Tan, J., see Genin, A.L.

Tang Tang, J., see Hejazi, W.

Taussig, M., see Nippa, M.

Tian, L., Tse, C.H., Xiang, X., Li, Y., Pan, Y., Social movements and international business activities of firms, 1200

Tietze, S., Cross-cultural management revisited: A qualitative approach, 1893

Tippmann, E., see Reuber, A.R.

Tippmann, E., see Stendahl, E.

Tong, T.W., see Prud'homme, D.

Tresl, J., see Hanousek, J.

Treviño, L.J., see Dheer, R.J.S.
Treviño, L.J., Doh, J.P., Internationalization of the firm: A discourse-based view, 1375

Tse, C.H., see Tian, L.

Uggerslev, K.L., see Field, J.G.

van Witteloostuijn, A., see Brakman, S.

Verbeke, A., The JIBS 2020 Decade Award: Unraveling the effects of cultural diversity in teams: A meta-analysis of research on multicultural groups, 1

Verbeke, A., The long-term energy transition and multinational enterprise complexity: A BJM-JIBS Joint Initiative, 803

Vyas, D., see Hope, O.-K.

Wang, D., Weiner, R.J., Li, Q., Jandhyala, S., Leviathan as foreign investor: Geopolitics and sovereign wealth funds, 1238

Wang, D., Zhu, Z., Chen, S., Luo, X.R., Running out of steam? A political incentive perspective of FDI inflows in China, 692

Wang, G., see Li, J.

Wang, W., see Hejazi, W.

Wang, Z., see Deng, Z.

Weiner, R.J., see Wang, D.

Westjohn, S.A., Magnusson, P., Peng, Y., Jung, H., Acting on anger: Cultural value moderators of the effects of consumer animosity, 1591

Wood, G., see Doh, J.

Wright, M., see Sun, P.

Xiang, D., see Kozlenkova, I.V.

Xiang, X., see Tian, L.

Yeung, B.Y., see Dau, L.A.

Zhang, N., see Clougherty, J.A.

Zhao, Y., Parente, R., Fainshmidt, S., Carnovale, S., MNE host-country alliance network position and post-entry establishment mode choice, 1350

Zheng, Y., see El Ghoul, S.

Zhou, K.Z., see Jean, R.-J.B.

Zhu, Z., see Wang, D.

\section{KEYWORD INDEX TO VOLUME 52}

abduction 1695

access to finance 321

administrative affiliation 621

agency theory 560

Airbnb 1445 
Alibaba 1501

alliances 545

allocentrism 78

anomic mindset 1695

arbitrary inefficiencies 1724

auditing 718

authenticity 245

B2B E-platform 1501

behavioral theory 1047

best practices 1229

bifurcation bias 148

big data 1797

born globals 1665

brand credibility 1559

budget allocation 105

bureaucracy 692

business and the environment 807

business groups 161

business history 971

business models 824, 1665

business/government interaction and relationships 454

Canada 306

capital structure 121

case study 282, 409, 971

central and eastern Europe 1750

central role of marketing 1429

change 409

chief marketing officer 363

China 595, 692, 773

Chinese high-speed train 621

climate change 904

collaborative consumption 1445

consumer animosity 1591

consumer behavior 1591

consumption convergence 105

content analysis 879

control 409

cooperation 1616

coordination 56

corporate governance in emerging markets 621

corporate political activity 1818

corporate social responsibility 525

corruption 321,1750

country selection 1279

country-of-origin 479

country-of-origin export intensity 504

COVID-19 1215,1871

craft 245

creditor rights 121 cross-border mergers and acquisitions 454, 1724

crosscultural experiments 1591

cross-cultural research/measurement issues 1797

cross-national 1871

crowdsourcing 1445

cultural differences 56, 245

cultural diversity 4

cultural industries 245

cultural intelligence 432

cultural intermediaries 245

cultural values 1591, 1871

culture $4,45,1529$

Decade Award 4, 45

decentralization 1279

deceptive signal 1096

decision-making under uncertainty 388

diaspora ownership 671

difference-in-differences 692, 1200

digital globalization 1646

digital platform 1096

discouraged borrowers 321

discourse-based analysis 1375, 1394, 1407, 1417

discretion 363

diversity 45

divestment 971

downside risk 388

dynamic endogeneity 339

dynamic MNE behavior 1047

econometrics 525

economic development 545, 746

ecosystems 1646

electoral systems 1775

emerging economies 545, 560, 718, 1529, 1854

emerging market agricultural sector 646

emerging market firms 671

emotion 1591

energy 807, 824, 853, 904

energy transition 930

entrepreneurship 746

ESG capabilities 1256

establishment mode 1350

ethnocentric 1695

ethnography 1695

expatriate 78, 1331

expatriate management 432

export growth strategy 1501

export intermediaries 245

export performance 1616

exporting 1096, 1429

externalities 999 
family firm internationalization 148, 1159

financial market development 1121

financial performance 504

financial statement certification 718

financial systems 121

firm performance 1429

firm value 212

firm-specific advantages 148, 824

fixed effects estimators 339

flexibility 409

foreign affiliate performance 1854

foreign CEO 525

foreign direct investment (FDI)

$306,692,930,1256,1750$

foreign investors 212

foreign market entry 1628

foreign R\&D headquarters 1302

formal institutions 1121

FSA-CSA matrix 930

FSAs 545

generalized inefficiencies 1724

generalized method of moments 339

geographic proximity 1302

gig economy 1445

global consumer behavior 105

global environment 807

global leadership 1695

global mindset 1695

global projects 56

global strategy 1031

global teams 4, 56

global value chains 1215

globalization 525, 1031, 1559, 1695

GLOBE cultural variables 1474

government 1238

government connections 321

government procurement 718

government stringency 1871

grand challenges 951, 999

headquarters-subsidiary relationship 409, 1069

historical method 1417

history 773

host country language proficiency 78

host-country expansion 1350

hypernorms 1229

IB paradigm 1365

ideologies 282

immigrants 504

immigration policies 432

import 1200 incentives 930

individual-level research 1365

industrial policy 853

industry-science linkages 1302

informal institutions 1121

informal pressures 1200

in-group collectivism 432

innovation 545

innovation and R\&D 621

innovation ecosystem 646

institutional arbitrage 1096

institutional changes 1256

institutional context 1375

institutional development 161

institutional environments 1501

institutional inefficiency 1724

institutional investors 212

institutional theory $321,621,853,879,1529$

institution-based view 1854

institutions 773, 951, 1724

instrumental variables estimation 671

intellectual property rights (IPRs) 773

interdependence 1616

interdisciplinary research 1394

intergovernmental ties 1279

internalization theory $161,363,824,1665$

international business 999

international business field 1394

international business research 339

international business theory 904, 1159

international corporate governance 212

international entrepreneurship 1665

international joint ventures 595

international marketing 1429, 1474

international NGO activist campaigns 971

international technology licensing 671

internationalization 161, 363, 479, 824, 1069, 1628

internationalization process 1096, 1394, 1407

internationalization theories and foreign market entry 1047, 1375, 1417

invention performance 560

investment 1775

investment horizon 212

Japan 245

jurisdiction shopping 853

knowledge 1724

knowledge sharing 432

knowledge transfer 1331

labor markets 1215

learning by doing 1047 
level of analysis 1365

liability of foreignness 282, 525, 1238

liability of origin 282, 671

literature review 23

location choice 1256

location strategy 853

location-bound/non-location-bound 824

logistic regression 1238

long-term energy transitions 807, 951

longitudinal case study 646

loose coupling 1646

macro-processes 773

managerial autonomy 560

managerial cognition 1695

managerial decision-making 1474

market concentration 105

market entry 1429

market share 105

matched sample 671

meso economics 161

meta-analysis 4, 23, 1591, 1797

methodological rigor 1365

methodological trends 1365

methods diversity 1365

metrics 1474

microfoundation 363

micro-processes 773

middle income trap 161

MNE-host-country relationships 454, 1238

MNEs and economic growth 306

mobile payments 1529

mobile wallets 1529

multi-level analysis 432

multinational corporations (MNCs) and enterprises

(MNEs) 388, 409, 545, 807, 904, 1031, 1069,

1750,1818

multinational networks 388

multinationals 999, 1775

network brokerage 1350

network centrality 1350

network effects 1529

networks 479,1628

new institutional economics 746

new internalization theory 148, 1646

nonlinear effects 1616

non-market capabilities 1256

nonmarket strategy 853, 1818

novel coronavirus 1871

oil and gas 879

open science 1797 organizational culture 1474

organizational embeddedness 432, 1331

organizational identity 1069

organizational learning 306

organizational stigma 282

panel data Tobit regression 504

patent 595

peace agreements 1256

peer-to-peer 1445

perceived brand globalness 1559

perceived brand localness 1559

pharmaceutical and biotechnology industry 1302

philosophy of science 1229

platforms 1529, 1646

PLS 1616

policy-oriented studies 454

political capabilities 1256

political constraints 560

political incentives 692

political relationships 1238

political rent seeking 161

political uncertainty 1775

politics 282

pollution havens 930

positive peace 1256

primary data 1365

private firms 718

privatization 1121

problemistic search 1047

process 479

product adaptation 245

product design 245

product market performance 121

productivity 306

pro-market reforms 1854

psychic distance 1616

public private networks 646

public sector organizations 646

qualitative research 479, 1069

quantitative review 23

rapid growth 1031

real option theory 1750

real options logic 388

recombination 148,545

regression analysis 746

regression with Heckman's two-step

corrections 1724

relationship management 1429

relationships 1628

renewable energy 879 
repatriation 1331

replication 1031

research and development (R\&D) 545, 595

research collaboration 1302

research ethics 1229

research methodology 23

rhetorical (de)legitimation 971

risk 1215

roles 56

scaling 1031

scripts 56

secondary data 1365

sharing economy 1445

signaling theory $671,1501,1559$

simulations 339

sister cities 1279

small-and-medium-sized enterprises (SMEs)

245,321

social categorization 78

social embeddedness 504

social identity perspective 525

social movements 1200

social networks 1200

SOE restructuration 621

South Africa 971

specific investments 1616

speed 1628

sponsored listing 1096

stakeholder-based view (SBV) 773 state-owned enterprises 560, 595, 930, 1121, 1238

strategic corporate social responsibility 1818

structural equation modeling 1559

subnational environments 671

subnational location choice 1279

survival analysis 1665

sustainability 807, 879, 904, 999

Sustainable Development Goals (SDGs) 999

synthesis 23

systematic review 1159

systemic change 951

teams and teamwork 45

technological catch-up 646

topics 1628

trade agreements 746

trade credit 1121

trade integration 746

trait activation theory 432

transaction costs 161

transnational entrepreneurship 504

two-sided markets 1529

two-stage instrumental variable approach 504

uncertainty 1750

uncertainty avoidance 78

United States 773

Uppsala model 1407, 1417, 1665

vicarious learning 1047

Publisher's Note Springer Nature remains neutral with regard to jurisdictional claims in published maps and institutional affiliations. 